\title{
Distributed Holistic Clustering on Linked Data
}

\author{
Markus Nentwig $^{12}$, Anika Groß $\aleph^{1,2}$, Maximilian Möller ${ }^{1,3}$, and Erhard Rahm ${ }^{1,2}$ \\ 1 Database Group, University of Leipzig \\ 2 nentwiglgross|rahmeinformatik.uni-leipzig.de \\ $3 \mathrm{~m}$.moellerestudserv.uni-leipzig.de
}

\begin{abstract}
Link discovery is an active field of research to support data integration in the Web of Data. Due to the huge size and number of available data sources, efficient and effective link discovery is a very challenging task. Common pairwise link discovery approaches do not scale to many sources with very large entity sets. We here propose a distributed holistic approach to link many data sources based on a clustering of entities that represent the same real-world object. Our clustering approach provides a compact and fused representation of entities, and can identify errors in existing links as well as many new links. We support a distributed execution of the clustering approach to achieve faster execution times and scalability for large real-world data sets. We provide a novel gold standard for multi-source clustering, and evaluate our methods with respect to effectiveness and efficiency for large data sets from the geographic and music domains.
\end{abstract}

\section{Introduction}

Linking entities from various sources and domains is one of the crucial steps to support data integration in the Web of Data. A manual generation of links is very timeconsuming and nearly infeasible for the large number of existing entities and data sources. As a consequence, there has been much research effort to develop link discovery (LD) frameworks [16] for automatic link generation. Platforms like dat ahub. io and sameas . org or repositories such as LinkLion [17] and BioPortal [21] collect and provide large sets of links between numerous different knowledge sources. They are valuable resources to improve the availability and re-usability of links in applications and avoid an expensive re-determination of the links. However, existing inter-source mappings can be incomplete and so far many sources are not interrelated at all.

Due to the huge number and size of available knowledge sources, link discovery is still a very challenging task. It is particularly complex to ensure high link quality, i.e., the generation of correct and complete link sets. Existing link repositories cover only a small number of inter-source mappings and automatically generated links can be erroneous in many cases $[4,19]$. Despite the huge number of sources to be linked, most LD tools focus on a pairwise (binary) linking of sources. However, link discovery approaches need to scale for n-ary linking tasks where more than two sources need to be matched, as well as for an increasing number of entities and sources that are added to the Web of Data over time [22].

To address these shortcomings we recently proposed an approach to cluster linked data entities from multiple data sources into a holistic representation with unified properties [15]. The method combines entities that refer to the same real world object in one 
cluster instead of maintaining a high number of binary links for $k$ sources. The approach is based on existing owl : sameAs links and can deal with entities of different semantic types as they occur in many sources (e. g., geographical datasets contain various kinds of entities such as countries, cities, lakes). It can further eliminate wrong links from existing link sets and identifies many new links, e. g., for previously unconnected sources. The clustered representation of entities with their properties and a cluster representative is more compact and comprehensive, allows central maintenance and access, and facilitates the integration of new data sources and entities. The clustering-based approach complements existing link discovery approaches to provide a compact and fused representation of entities. Initial clusters can further serve as a basis for an incremental extension of clusters by newly added entities as outlined in [22].

Considering the huge size and number of sources to be linked, scalability becomes a major issue. Workflows for linking and/or clustering of entities usually comprise several complex phases such as similarity computation to find similar entities or clusters that need to be merged. Therefore, a distributed realization of holistic clustering approaches for $k$ data sources would be valuable to improve its efficiency. In particular, complex operations should be executed in parallel in a distributed environment in order to reduce execution time and to enable scalability for large real-world datasets. Big Data frameworks like Apache Spark [25] or Apache Flink [2] provide fast execution engines to process very large datasets in a distributed environment. These generic frameworks allow to define complex data processing workflows using common operators like map or join as well as user-defined functions. So far there has been only few related work considering distributed clustering for linked data and data integration workflows. With regard to the ever increasing amount of data that needs to be linked and integrated in typical big data processing workflows, it is essential to develop scalable solutions for link discovery and holistic entity clustering.

A further and long-standing problem is the poor availability of reference datasets that can be used as gold standard for quality evaluations. Since LD tools usually focus on pairwise matching, the few existing benchmarks cover links between two data sources. However, there is an increasing need to evaluate the effectiveness of multisource clustering, as holistic data integration scenarios like the construction of knowledge graphs gains increasing research interest $[3,20]$. The creation of new reference datasets for quality evaluation of n-ary linking and clustering approaches can be useful for the community and support the development of improved holistic clustering approaches.

In this work, we study distributed holistic clustering as well as its evaluation on real-world data from different domains. We make the following major contributions:

- We present a distributed holistic clustering approach for linked data to enable an effective and efficient clustering of large entity sets from many data sources. The implementation is based on the distributed data processing system Apache Flink.

- We provide a novel reference dataset for multi-source clustering from the geographic domain. We evaluate the effectiveness of our approach with the new gold standard and a further artificial dataset from the music domain.

- We further evaluate the efficiency and scalability of the distributed holistic clustering for very large datasets with millions of entities from the two domains. 
The remainder of the paper is organized as follows. We first introduce preliminaries w.r.t. link discovery and the holistic clustering approach (Section 2). In Section 3 we present the implementation of the distributed holistic clustering. We present the clustering gold standard in Section 4.2 and show evaluation results in Section 4. Finally, we discuss related work in Section 5 and conclude in Section 6.

\section{Preliminaries}

\subsection{Link Discovery}

Link discovery (LD) has been studied intensively and there is a large number of approaches and prototypes available [16]. LD tools usually identify owl : sameAs links between entities that denote the same real-world object. However, link discovery might also include the identification of links with other semantics such as the association $\mathrm{dbp}: \mathrm{birthPlace}$ between a person and a city. Entities are described by a unique URI, e.g., http://dbpedia.org/page/Leipzig, and can have different semantic types, e. g., dbo: Country or dbo: City for the geographic domain. Each entity can be further described by specific properties from ontologies such as $r \mathrm{dfs}: l$ abel. The matching process usually covers the computation of similarity values for pairs of entities, and candidate links are subsequently filtered by selection strategies based on the link similarity. The herein presented approach complements existing binary LD approaches for linking two data sources by allowing for a distributed holistic clustering of entities from $k$ different data sources $S$. Same-as links created via binary LD constitute a mapping $M_{i, j}=\left\{\left(e_{1}, e_{2}, \operatorname{sim}\right) \mid e_{1} \in S_{i}, e_{2} \in S_{j}, \operatorname{sim}[0,1], i \neq j, 1 \leq i, j \leq k\right\}$. We reuse existing mappings between different data sources $\mathcal{M}=\bigcup_{i, j=1}^{k} M_{i, j}$ as input for the holistic clustering.

\subsection{Holistic Clustering Workflow}

In our previous work [15] we presented the holistic clustering workflow shown in Figure 1. Here we present the realization of the holistic clustering in a distributed environment for improved performance and scalability of the approach. We therefore use the distributed processing system Apache Flink as well as its graph-processing API Gelly. Our implementation is based on the property graph model. The input of the workflow is a graph $\mathcal{G}=(\mathcal{V}, \mathcal{E})$ with a set of vertices (entities) $\mathcal{V}=\{v\}$ with each vertex having an unique identifier and domain-specific properties, e.g., $v=\{$ id, label, source, type, $\ldots\}$, as well as a set of edges (links) $\mathcal{E}=\{e\}$ where each edge has a source and a target vertex id in $\mathcal{V}$. Edges may also have additional properties like a similarity value. The input graph $\mathcal{G}$ is a similarity graph constructed based on an existing mapping of input links $\mathcal{M}$, e. g., from an publicly available link repository. For our representation of clusters we define a set of clusters $\mathcal{C}=\{C\}$ where each $C \in \mathcal{C}$ has a cluster id $c_{\text {id }}$, a set of contained vertex ids from $\mathcal{V}$ and a cluster representative $r$ containing unified properties like a label, a semantic type and the original data sources, e.g., $r=\left\{\right.$ label, $\left.\left\{v_{1}, v_{2}, \ldots\right\},\left\{S_{i}, S_{j}, \ldots\right\},\left\{t_{1}, t_{2}, \ldots\right\}, \ldots\right\}$. Although input links 


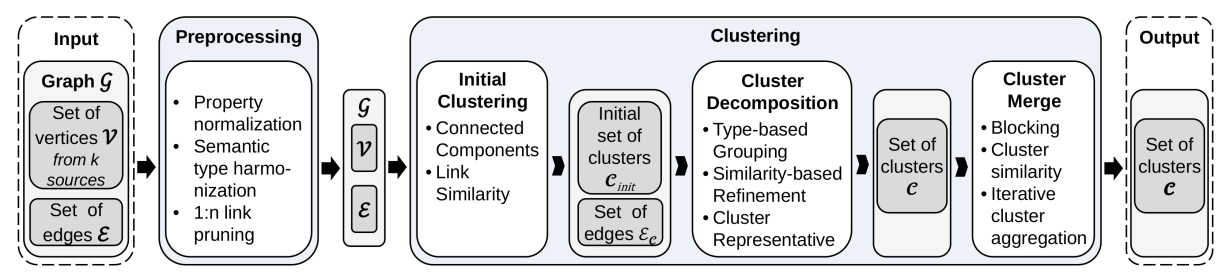

Fig. 1: Holistic Clustering Workflow

might be associated with similarity values, we compute new similarities between vertices within clusters (intra-cluster edges $\mathcal{E}_{\mathcal{C}}$ ) to enable the use of sophisticated measures and to ensure comparability of link similarities within our workflow.

The basic holistic entity clustering [15] employs a four-phase approach (see Figure 1). During Preprocessing we normalize properties for vertices and harmonize their semantic types. The harmonization is necessary since semantic type information can differ substantially between different sources, and might also be missing. We therefore use background knowledge about type equivalences and comparability to harmonize original types to more general ones, e. g., city, town and suburb refer to the more general type settlement. For duplicate-free data sources there should be at most one equivalent entity in another data source. For entities with several links to entities from the same source (1:n links), we therefore only keep the best link for the considered entity in order to ensure the one-to-one cardinality. As an Initial Clustering we compute connected components for the input graph $G$. Within each initial cluster $C \in \mathcal{C}$ we then compare all covered vertices based on different similarity measures to obtain a set of intra-cluster edges $\mathcal{E}_{C}$ and similarity values for the subsequent steps. In the Cluster Decomposition phase we refine the clusters by splitting them according to semantic type information and edge similarities within the cluster. Each cluster should only contain vertices of the same semantic type, and edges within a cluster have to exceed a certain similarity threshold. Vertices that do not fulfill these criteria constitute a new cluster. For each cluster we create a cluster representative by combining property and data source information from the covered vertices. The final Cluster Merge phase aims at combining smaller but similar clusters. Therefore, we first compute similarities between different clusters, and then iteratively merge the best matching cluster combinations, respectively, checking constraints like the maximal possible cluster size $k$ and already covered data sources within a cluster. In the here presented implementation we further employ a new blocking step prior to the matching of the cluster representatives in order to reduce the search space and avoid unnecessary comparisons.

In contrast to [15], this paper outlines a distributed implementation of the holistic clustering approach as well as a comprehensive evaluation of its quality and efficiency.

\section{Implementation of Distributed Holistic Clustering}

In this section we outline the workflow and implementation for our distributed holistic clustering approach based on the big data stream and batch processing system Apache Flink [2]. We first introduce the used transformation operations from Apache Flink as 
well as graph datasets provided by Flink's graph processing engine Gelly (Section 3.1). We then present the transformation and adaptation of the holistic clustering approach towards a distributed processing workflow using Apache Flink/Gelly (Section 3.2).

\subsection{Apache Flink and Gelly API}

For our approach we make use of the batch processing part of Apache Flink. It provides the DataSet API and well-known dataset transformations like filter, join, union, group-by or aggregations (relational databases) and map, flat-map and reduce (MapReduce paradigm). Special in-memory, distributed data structures called DataSets store data within Flink programs. DataSets can be manipulated based on so called transformations that return a new DataSet. Some transformation operations make use of user-defined functions (UDFs) and allow for customized definitions how DataSet values need to be changed. Flink optimizes the execution of succeeding transformations, employs lazy evaluation and avoids intermediate materialization of results. A set of libraries provides additional functionality for Flink such as complex event processing, graph processing or machine learning. We will make use of the graph processing engine (Gelly) in our holistic clustering workflow. In particular, we employ Gelly graphs containing a DataSet of vertices and edges

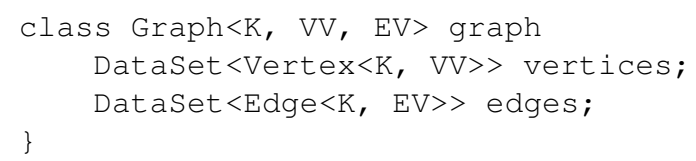

The complex data types Vertex and Edge are inherited from the Flink Tuple classes Tuple $2<K, V V>$ (type $K$ as vertex id, VV as vertex value), Tuple $3<K, K, E V>$ (source vertex id, target vertex id (each type $\mathrm{K}$ ) and $\mathrm{EV}$ as edge value), respectively. Operators like join, filter or group-by rely on tuple positions (starting from 0), e. g.,

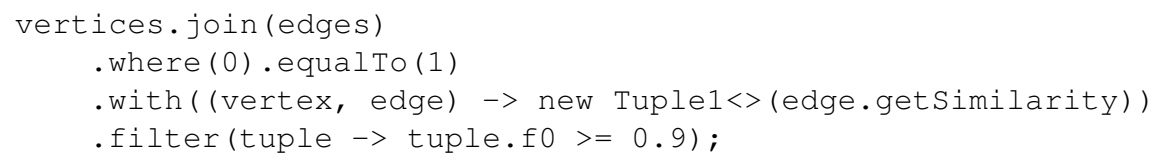

will join all edges with the vertices where the vertex id (position 0 in vertices) equals the target id of the edge (position 1 in edges) and returns the similarity value if the accompanied filter function is evaluated and returns true. Please see Table 1 for more detailed explanations on transformations that we use within our approach. Depending on the physical data distribution, data needs to be shuffled across cluster nodes to execute transformations such as join or group-reduce requiring a reorganisation of data w.r.t. the used (grouping) key. Strategies like repartition/map-side join or groupreduce/combine can be applied to reduce the necessary network traffic.

Besides the used graph data model we benefit from Flink's and Gelly's abstract graph processing operators like graph neighborhood aggregations or abstracted models for iterative computations. In particular, we will make use of the Flink delta iteration in different variations, e.g., vertex-centric iteration (Pregel [12]), gather-sum-apply computation (PowerGraph [5]) or custom implementations for delta iterations as discussed in the following sections. 


\begin{tabular}{|c|c|}
\hline Operator & Description \\
\hline (Flat)Map & $\begin{array}{l}\text { Map- and FlatMap transformations apply a UDF on each element of the } \\
\text { DataSet. Map functions emit exactly one resulting element per input while } \\
\text { FlatMap functions may emit arbitrary result elements (including none). } \\
\text { input.map (udf: IN }->\text { OUT) }\end{array}$ \\
\hline Filter & $\begin{array}{l}\text { Return all DataSet elements for which the UDF returns true. } \\
\text { input.filter (udf: IN }->\text { Boolean) }\end{array}$ \\
\hline (Flat)Join & $\begin{array}{l}\text { A Join transformation combines elements of two DataSets with equal values on } \\
\text { (tuple) key(s) and creates exactly one result element for Join and arbitrary result } \\
\text { elements for the FlatJoin based on a UDF. } \\
\text { leftInput.join (right Input). where (leftKeys) } \\
\text {. equalTo(rightKeys). with (udf: (left, right) -> OUT) }\end{array}$ \\
\hline
\end{tabular}

ReduceGroup Elements in a DataSet can be grouped on keys of the DataSet fields. Each group executes a user-defined group-reduce function.

input.groupBy (keys). reduceGroup (udf: IN $\rightarrow$ OUT)

Aggregate Aggregations like sum, min, max or UDFs can be applied to grouped DataSets input.aggregate (SUM, key);

Table 1: Apache Flink DataSet transformations used for holistic clustering approach.

\subsection{Holistic Clustering}

In this section, we will discuss the transformation and adaptation of the holistic clustering workflow towards a distributed processing workflow in Apache Flink. From a high-level perspective, we read input vertices and edges into a Gelly graph and apply a set of transformation operators to generate entity clusters. Intermediate results are represented as Gelly vertices, edges and graphs and can be written to disk, e. g., as JSON. Within complex transformations we remove unneeded properties using Flink TupleX representation instead of Vertex or Edge to reduce the amount of network traffic and memory consumption. We illustrate the workflow steps using the running example in Fig. 2. There are six input edges $\mathcal{E}$ and seven input vertices $\mathcal{V}$ further described by a label $\left(l_{1}, l_{2}, \ldots\right)$, the originating data source from $S$ and colored dependent on their semantic type ( $t 1, t 2$ or no type).

Preprocessing During preprocessing we apply several user-defined functions on the input graph, e. g., to harmonize semantic type information, remove inconsistent edges and vertices and normalize the label property value. First, we compute similarities only for given input edges based on vertex property values. For each vertex, we carry out a consistency validation using grouping on adjacent vertices and associated edges, and further remove neighbors with equal data sources (for details see [15]). For vertices with multiple semantic type values we ensure that grouping on the type supports overlapping type information for later processing. For clarity reasons, we do not use multiple types in our running example. We also omit the preprocessing step in the example (Fig. 2) and directly start with the preprocessed input graph $\mathcal{G}=(\mathcal{V}, \mathcal{E})$. 


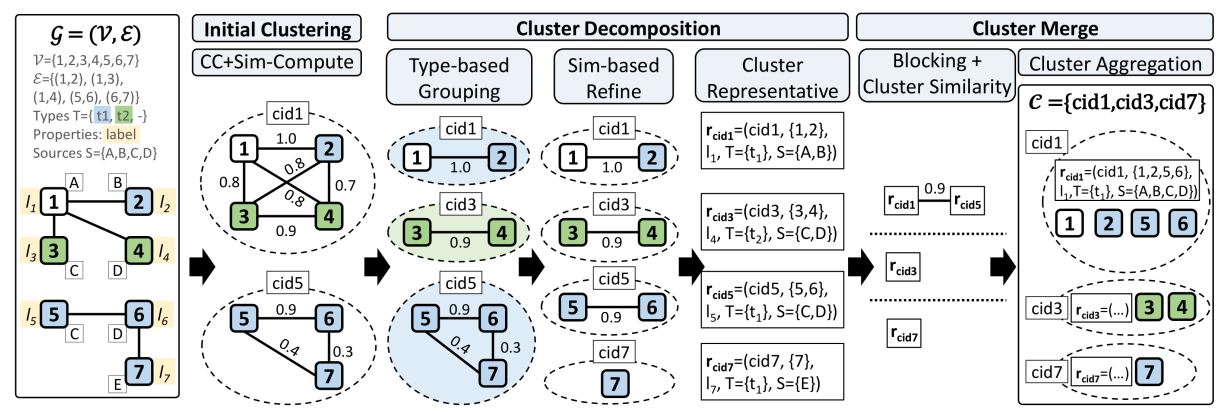

Fig. 2: Example clustering workflow.

Initial Clustering To determine initial clusters, we use a gather-sum-apply operator to determine the connected components (CC) within $\mathcal{G}$ and assign a cluster id to each vertex. In the example, vertices 1-4 obtain obtain cluster ids cidl and vertices 5-7 cid5. With these cluster ids, we generate all missing links within each cluster. We therefore use a CoGroup function on cluster ids of all vertices, combine vertices within clusters to get intra-cluster edges $\mathcal{E}_{\mathcal{C}}$ and compute similarities for these edges. We apply a combination of similarity measures on different properties such as a linguistic similarity on labels and other properties and possibly further domain-specific measures like a normalized geographical distance.

Type-based Grouping Type-based grouping is the first part of the decomposition phase to split clusters into sub-components dependent on the compatibility of semantic types. Fig. 3 a shows the sequence of applied transformations and short descriptions. First the initially clustered vertices are grouped on their cluster ids. Each cluster group executes a ReduceGroup function to assign new cluster ids based on different semantic type, e. g., in the example vertex 3 and 4 are separated from vertex 2 . All vertices without type (like vertex 1) require a special handling. We apply GroupReduceOnNeighbors (a Gelly CoGroup function to handle neighboring vertices and edges for each vertex) to produce Tuple 4 objects for vertices with missing semantic type. In our example, vertex 1 creates a Tuple $4<>$ (id, sim, type, cid) for each of its outgoing edges $((1,2),(1,3),(1,4))$, namely Tuple $4<>\left(1,1.0, t \_1, \mathrm{cid} 1\right)$ for edge $(1,2)$ and Tuple4<>(1, $\left.0.8, t_{-} 2, c_{i d 2}\right)$ for edge $(1,3)$ and $(1,4)$. Now, grouping on the vertex id executes an aggregation function for each group to return the tuple with the highest similarity per vertex, which is $\operatorname{Tup} l e 4<>\left(1,1.0, t \_1\right.$, cidl $)$ for vertex 1 in our running example. Finally, the vertices from the initial group-by (see Fig. 3 a are joined with the remaining Tuple 4 objects to update vertices without semantic type with their new cluster id, e.g., vertex 1 is assigned to cluster cid1. The result of the type-based grouping is a set of clusters with intra-cluster edges.

Similarity-based Refinement We further decompose clusters by removing non-similar entities that do not match with other entities in their cluster. This part is realized using the Gelly vertex-centric iteration. Basically, the implemented vertex-centric itera- 
(a)

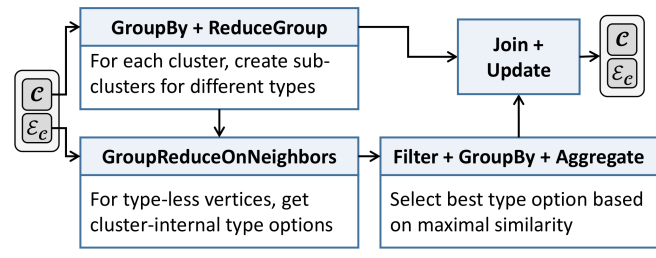

(b)

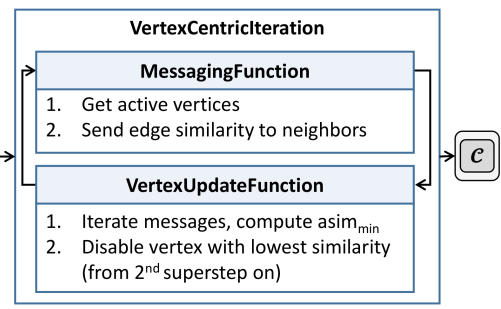

Fig. 3: Sub-workflows with operators for type-based grouping (a) and similarity-based refinement (b).

tion switches between a custom MessagingFunction and a VertexUpdateFunction (see Fig. $3 \mathrm{~b}$ for details), an iteration round is called superstep. In the first round, all vertices are active and therefore send messages to all their neighbors. Messages are Tuple 3 objects containing the originating id, the edge similarity and an average edge similarity asim over all incoming messages (which is 0 in the first iteration). Starting with the second iteration, we illustrate the sent messages for vertex 7 in cluster cid5 in our example: vertex 5 sends Tuple $3<>(5,0.4,0.65)$ to 7 , vertex 6 sends Tuple $3<>(6$, $0.3,0.6)$ to 7 and vertex 7 sends messages to 5 and 6 , resulting in the average similarity asim $=(0.4+0.3) / 2=0.35$ for vertex 7 . Now in each cluster the vertex with the lowest asim will be deactivated (and is therefore excluded from the cluster) given that this asim is below a certain similarity threshold. In our example in Fig. 2, vertex 7 with asim $=0.35$ will be deactivated and isolated into cluster cid7. The vertex-centric iteration terminates based on these criteria: First, vertices only send messages when their own vertex value was updated in the current round. Second, all deactivated vertices will never send messages again. Lastly, a maximum number of iteration rounds can be set.

Cluster Representative As final step in the decomposition phase, we create a representative for each cluster. Therefore, a GroupReduce function based on the cluster id is used to combine property values to a unified representation. To determine the cluster representative, we aggregate cluster information about the covered data sources, semantic types and contained vertices and select the best values for certain properties such as label or geographic coordinates using the GroupReduce on clusters. Detailed examples for representants are shown in Fig. $2\left(r_{c i d 1}, r_{c i d 3}, r_{c i d 5}\right.$ and $\left.r_{c i d 7}\right)$.

Cluster Merge For the final merge phase in our distributed holistic clustering workflow we use the Flink DeltaIterate operator together with user-defined functions. The main operators are sketched in Fig. 4. During merge, we iteratively aggregate highly similar (likely small) clusters into larger ones. With the creation of representatives for each cluster, we already reduced the amount of entities to handle in the merge step. Due to the fact that we potentially compare every cluster with every other cluster, the quadratic complexity can become a problem for very large cluster sets. Thus we employ blocking strategies and avoid unnecessary comparisons. Currently we implement standard blocking on specified property values such as using the first letters of the label 


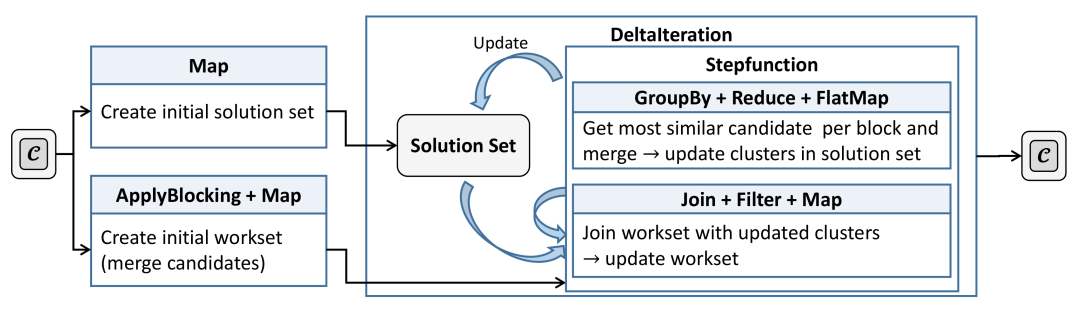

Fig. 4: Sequence of transformations for the cluster merge using Flink Deltalteration

as blocking key. We avoid further unnecessary comparisons since we do not compare representatives with incompatible types based on semantic type and check for already covered data sources since we assume duplicate-free data sources. In our example in Fig. 2 three blocks are shown for the blocking: $r_{c i d 1}$ and $r_{c i d 5}$ need to be compared, such that a triplet $\left(r_{c i d 1}, 0.9, r_{c i d 5}\right)$ is created as a merge candidate. For $r_{c i d 3}$, no merge candidate is created because there is no other representative with type $t_{2}$, whereas $r_{c i d 7}$ is in a separate block due to its dissimilar label compared to other representatives.

The delta iteration is started with an initial solution set containing the previously determined clusters and an initial workset (merge candidates) as seen in Fig. 4. Within each iteration of DeltaIterate, a custom step function works on the current workset to update the workset and to generate changes on the current solution set. The next workset and the updates to the solution set are then passed to the next iteration step. In detail, the workset is grouped by the blocking key and for each block the triplet with the highest similarity exceeding the minimum similarity is selected using a custom Reduce function. For our running example, $\left(r_{c i d 1}, 0.9, r_{c i d 5}\right)$ is the best merge candidate and therefore merged with a custom FlatMap function. The new cluster $r_{c i d 1}$ contains combined values for properties like sources $S=\{A, B, C, D\}$, the list of contained vertices $(\{1,2,5,6\})$ and unified properties like the label $l_{1}$. This will directly affect the cluster representatives in the solution set, and the already merged cluster $r_{c i d 5}$ will be deactivated in the solution set. Now the merge candidates within the workset are adapted based on changed clusters within the iteration step (see Fig. 4 solution set). Deactivated cluster representatives are replaced by the appropriate new cluster (here $\left.r_{c i d 1}\right)$, and merged triplets $\left(\left(r_{c i d 1}, 0.9, r_{c i d 5}\right)\right)$ as well as generated duplicate triplets are removed from the workset. Again, triplets are discarded if the data sources for the participating clusters overlap or exceed the maximum possible number of covered sources. The delta iteration ends either when the workset is empty (default, and true for our running example after the first iteration) or a maximum number of iterations took place. Note, that for larger datasets, parts of the dataset will converge faster to a solution, when clusters can not be merged anymore. These parts will not be recomputed in following iterations, such that only smaller parts of the data will be handled. 


\section{Evaluation}

In the following we evaluate our distributed holistic clustering approach w.r.t. effectiveness and efficiency for datasets from the geographic and music domains. We first describe details of the used datasets (Section 4.1). We then present a novel reference dataset for multi-source clustering and describe its creation process (Section 4.2). Based on this real-world and a further artificial reference dataset, we evaluate the effectiveness of our approach (Section 4.3). Based on large datasets from the geographic and music domains we also analyze the efficiency and scalability of the distributed holistic clustering approach.

\subsection{Datasets}

To evaluate the distributed holistic clustering, we use five datasets of different sizes from the music and geographic domains. Table 3 shows the available property values for entities, the number of covered entities and sources, as well as the number of correct links and clusters in the used reference mappings. Datasets DS1 and DS3 are used to evaluate the quality of entity clusters generated by the distributed holistic clustering while DS2, DS4 and DS5 are used to analyze the efficiency and scalability of our approach (see Section 4.3). In the following, we briefly describe the datasets for the two domains before we describe the novel reference dataset for multi-source clustering in Section 4.2 .

Geographic Domain We use two datasets (DS1, DS2) from the geographic domain, covering entities from the data sources DBpedia, GeoNames, NY Times, Freebase for DS1 and additionally LinkedGeoData for DS2. Entities for both datasets have been enriched with properties like entity label, semantic type, and geographic coordinates by using provided SPARQL endpoints or REST APIs. DS1 is based on a subset of existing links provided by the OAEI 2011 Instance Matching Benchmark $^{4}$ that is also a subset of DS2. Figure 5 shows the number of input entities and resulting numbers of links and clusters for the holistic clustering. The 3,054 entities in DS1 create a novel reverence dataset for multi-source clustering (see Section 4.2). Dataset DS2 covers about

\footnotetext{
${ }^{4}$ http://oaei.ontologymatching.org/2011/instance/
}

\begin{tabular}{lcccccc}
\hline domain & entity properties & dataset & \#entities & \#sources & \#correct links & \#clusters \\
\hline \multirow{2}{*}{ geography } & label, semantic type, & DS1 & 3,054 & 4 & 4,391 & 820 \\
& longitude, latitude & DS2 & $1,537,243$ & 5 & - & - \\
\hline \multirow{2}{*}{ music } & artist, title, album, & DS3 & 19,375 & 5 & 16,250 & 10,000 \\
& year, length, & DS4 & $1,937,500$ & 5 & $1,624,503$ & $1,000,000$ \\
& language, number & DS5 & $19,375,000$ & 5 & $16,242,849$ & $10,000,000$ \\
\hline
\end{tabular}

Table 3: Overview of evaluation datasets. Number of resulting clusters and deduced correct links are given for reference datasets. 

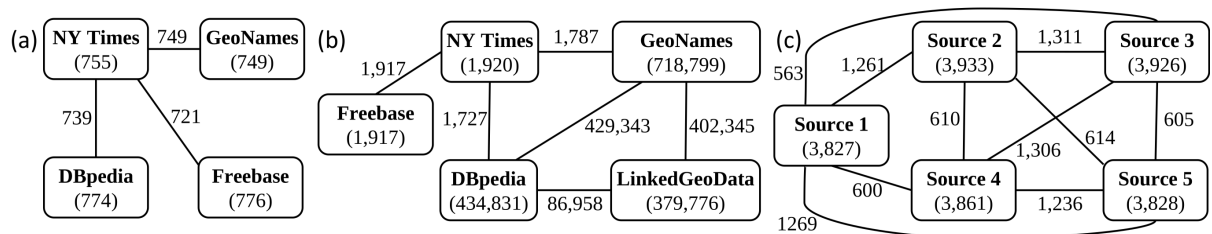

Fig. 5: Dataset structures for DS1 (a), DS2 (b) and DS3 (c) with number of entities and links.

1.5 million entities from five sources (see Fig. 5 b) and originates from the link repository LinkLion [17]. We reuse about 1 Mio existing ow 1 : sameAs links from LinkLion as input for the holistic clustering. However, there is no reference dataset available to evaluate the quality of created clusters for dataset DS2. We use DS2 to evaluate the scalability of our approach for very large entity sets.

Music Domain The publicly available Musicbrainz dataset covers artificially adapted entities to represent entities from five different data sources $[8]^{5}$. Every entry in the input dataset represents an audio recording and has properties like title, artist, album, year, language and length. The property values have been partially modified and omitted to generate a certain degree of unclean data and duplicate entities that need to be identified. This includes format changes for properties like year (e.g., '06, 06 or 2006) and song length $(2 \mathrm{~m} 4 \mathrm{sec}, 02: 04,124000$ or 2.0667).

The artificially generated datasets cover between 19,375 and 19,375,000 tuples (see Table 3, DS3 to DS5). Each of the datasets contains a fixed proportion for each cluster size: cluster size 1 (50\%), size 2 (25\%), size 3 (12.5\%), size 4 and 5 (6.25\%, resp.). This means, that for instance $12.5 \%$ of all entities are in clusters of size 3 . Beside a set of artificially created duplicates, each dataset covers cluster ids from which links between entities, that refer to the same object, can be easily derived. Resulting clusters cover between about 16,000 and 16,000,000 links and up to 10 million clusters. DS3 will be used for quality evaluation, while DS4 and DS5 are used to analyze the scalability of the distributed holistic clustering.

\subsection{Reference Dataset for Multi-source Clustering}

We created a new manually curated reference dataset for multi-source clustering to support the evaluation of holistic clustering approaches w.r.t. the quality of generated clusters. Available benchmarks usually only contain links between two data sources. We here provide a gold standard based on real-world data from the geographic domain and make it available for other researchers. The reference dataset covers the input dataset and the perfect cluster result as JSON files and can be downloaded at https://dbs.uni-leipzig.de/research/projects/linkdiscovery.

The reference dataset is a selection of entities with the semantic type "settlement" from the location subset of the OAEI 2011 Instance Matching Benchmark. We made a

\footnotetext{
${ }^{5}$ Musicbrainz test data https://vsis-www. informatik.uni-hamburg.de/oldServer/teaching//projects/
} 

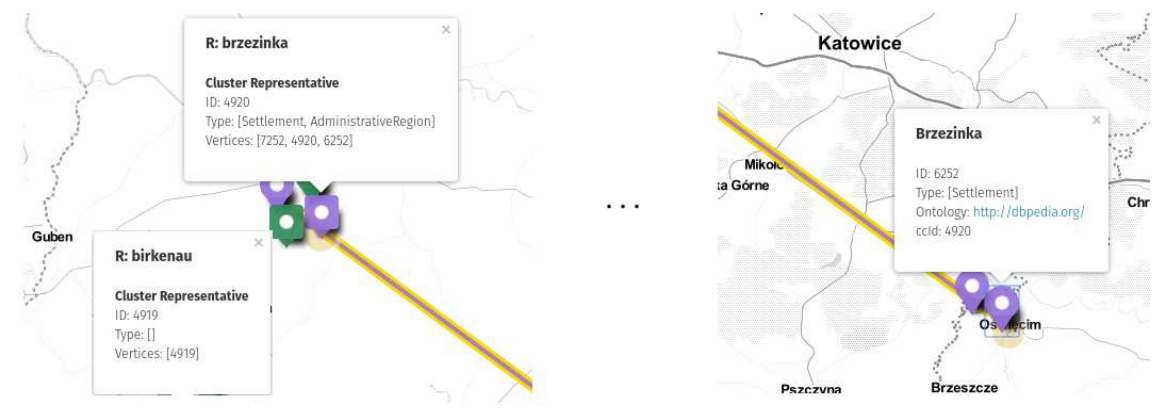

Fig. 6: Visualization of an incorrect cluster for two different settlements named "Brezinka" and "Birkenau" connected by an incorrect same-as link. Cluster representatives are illustrated by rectangles (on the left), while vertices are shown as circular pins (on the right).

manual selection decision for vertices using available properties and edges. We further checked the correctness of semantic types. For instance, the vertex for "Canary Island" was removed since its correct type in the geographic dataset should be "island" instead of "settlement". Removing a vertex resulted in the deletion of all its associated edges. For manual curation we visualized the data using an open-source geographic map tool ${ }^{6}$ (see Fig. 6). To determine the perfect clusters and check for the correctness of the input data, we used two views on the data. First, the original links are represented as (thick) yellow links. The second view shows the resulting clusters created by the holistic clustering approach. Colored circular pins represent actual vertices with their properties, while rectangular pins illustrate the newly determined cluster representatives with label, type, and vertices within the cluster. The pin color distinguishes different clusters.

In particular, correct clusters were defined based on the results of the holistic clustering. In case of doubt, Wikipedia was used as additional knowledge source about certain geographical entities. For each cluster, we decided whether it was correct or needs to be deleted, split into several clusters or merged with another cluster. During the manual curation, all clusters of maximum size (w.r.t. the number of data sources) were correct. Modifications to obtain the perfect result included the addition of further vertices to a cluster or removing vertices from clusters. For example in Fig. 6, the original Birkenau/Brzezinka cluster needs to be split into two clusters due to a wrong link between two distinct geographic places. In the original data the cluster covered all four entities. The holistic clustering determined two clusters. The green pin labeled Birkenau (Poland) by NYTimes, as well as the purple pin next to it (labeled Brzezinka by GeoNames) should actually form one cluster. The two vertices of the south-east cluster are another settlement in Poland which has the same name, Brzezinka. The example represents a difficult case for automatic clustering and linking due to very differing vertex labels. The resulting reference dataset covers 820 cluster representatives containing information on covered cluster vertices. Based on the cluster ids one can easily derive all intra-cluster links to obtain the set of all correct links.

\footnotetext{
${ }^{6}$ uMap https : / / umap.openstreetmap.fr/en/, import data as GeoJSON
} 


\begin{tabular}{|c|c|c|c|c|c|c|c|c|c|}
\hline & \multicolumn{3}{|c|}{ config 1} & \multicolumn{3}{|c|}{ config 2} & \multicolumn{3}{|c|}{ config 3} \\
\hline & $P$ & $\mathrm{R}$ & $\mathrm{F} 1$ & $P$ & $\mathrm{R}$ & $\mathrm{F} 1$ & $\mathrm{P}$ & $\mathrm{R}$ & $\mathrm{F} 1$ \\
\hline input links & 0.933 & 0.806 & 0.865 & 0.964 & 0.938 & 0.951 & 0.981 & 0.799 & 0.881 \\
\hline best (star1, star2) & 0.863 & 0.844 & 0.853 & 0.963 & 0.941 & 0.952 & 0.951 & 0.838 & 0.891 \\
\hline holistic & 0.903 & 0.824 & 0.862 & 0.913 & 0.919 & 0.916 & 0.968 & 0.836 & 0.897 \\
\hline
\end{tabular}

Table 4: Evaluation of cluster quality for geography dataset DS1 w.r.t. precision (P), recall $(\mathrm{R})$ and F-measure $(\mathrm{F} 1)$.

\subsection{Experimental Results}

We now present evaluation results w.r.t. the quality of the determined clusters as well as the scalability of the distributed holistic clustering for the five datasets DS1-DS5.

Setup and Configurations The experiments are carried out on a cluster with 16 workers, each of them equipped with a Intel Xeon E5-2430 6x 2.5 GHz, 48 GB RAM, 2x 4 GB SATA disks and 1 GBit ethernet connection. The machines operate on OpenSUSE 13.2 using Hadoop 2.6.0 and Flink 1.1.2. All experiments are carried out three times to determine the average execution time.

In order to obtain input link datasets to apply holistic clustering on the geographic dataset DS1, we applied LD methods on the input entities using three different configurations (config 1, config 2, config 3). All configurations compute similarities based on JaroWinkler on the entity label; configurations 2 and 3 additionally compute a normalized geographic distance similarity below a maximum distance of $1358 \mathrm{~km}$. Config 1 applies a minimal similarity threshold of 0.9 for labels while configs 2 and 3 apply threshold 0.85 and 0.9 for the average label and geographic similarity, respectively. Links for DS2 were extracted from the LinkLion [17] repository, and have been computed by different LD tools from the community.

For the music dataset $D S 3$ we created input links using a soft TF/IDF implementation weighted on title (0.6), artist (0.3) and album (0.1) with a threshold of 0.35. DS4 and DS5 are used to show scalability, therefore, we simply create edges based on the cluster id from the perfect result by linking the first entity of each cluster with all its neighbors.

Quality We now analyze the achieved cluster quality for the geographic dataset DS1 based on precision, recall and F-measure. We first use existing input links from the considered subset in the original OAEI dataset (see Fig. 5 a). This manually curated benchmark achieves a precision of $100 \%$. However, many links between certain data sources are missing leading to a reduced recall of only $50 \%$, and a F-measure of $66.7 \%$. With the holistic clustering approach, we achieve very good results w.r.t. recall $(97.1 \%)$ while preserving a good precision $(99.8 \%$ ) resulting in the F-measure of $98.5 \%$. This shows that our approach produces high-quality clusters based on existing input links thereby finding many new links.

However, as input mappings are not perfect in real-world situations, we used automatically generated input links for three linking configurations (config 1-3) as described 


\begin{tabular}{cccc}
\hline & $\mathrm{P}$ & $\mathrm{R}$ & $\mathrm{F} 1$ \\
\hline input links & 0.835 & 0.783 & 0.808 \\
holistic & 0.890 & 0.861 & $\mathbf{0 . 8 7 6}$ \\
\hline
\end{tabular}

Table 5: Evaluation of cluster quality for music dataset DS3 w.r.t. precision (P), recall (R) and F-measure (F1).

\begin{tabular}{ccccc}
\hline \#workers & pre & \multicolumn{4}{c}{ dec } & merge & total \\
\hline 1 & 312 & 668 & 351 & 1331 \\
2 & 164 & 367 & 268 & 799 \\
4 & 79 & 231 & 207 & 518 \\
8 & 45 & 130 & 186 & 361 \\
16 & 23 & 42 & 162 & 227 \\
\hline
\end{tabular}

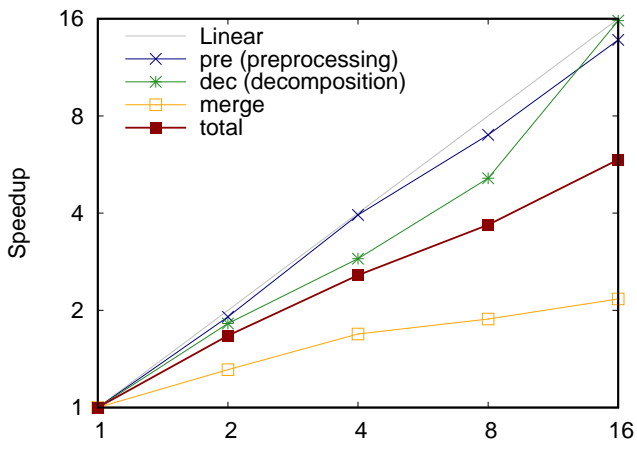

Fig. 7: Execution times (left) in seconds and speedup (right) for geographic dataset DS2 for the single workflow phases and total workflow.

above. To evaluate the cluster quality, we further compare our results with recently published results from [23]. The work implemented several existing clustering algorithms. Here, we only select the respectively best results achieved with two versions of Star clustering [1] (star1, star2). It is important to note that in contrast to our holistic clustering approach, star clustering creates overlapping clusters, thus clusters may contain duplicates. Besides, star clustering does not create a compact cluster representation. Table 4 shows results w.r.t. the cluster quality for the computed input links, the best result of (star1, star2) and our distributed holistic clustering approach. The holistic clustering (F-measure $86.2 \%$ ) nearly retains the input link quality $(86.5 \%$ ) for config 1 , while best(star1, star2) achieves slightly worst results. For config 2, the star2 implementation achieves a slightly better F-measure (95.2\%) compared to the input mapping $(95.1 \%)$. For config 3 the holistic clustering improves the quality of the input mapping by $1.6 \%$ w.r.t. F-measure (89.7\%).

For the music domain, we evaluate the cluster quality for DS3 using a set of computed input links (see setup in Section 4.3). Overall, the quality of the input links is lower than for DS1. Due to strongly corrupted entities and more properties, DS3 is more difficult to handle. Applying the holistic clustering, we identify a quality improvement for both precision and recall, resulting in a significant increase of F-measure by approx. $7 \%$ to $87.6 \%$ (see in Table 5) showing that our holistic clustering approach is able to handle such unclean data.

Overall, the holistic approach achieves competitive results although the DS1 dataset facilitates achieving relatively good input mappings making it difficult for any clustering approach to find additional or incorrect links. 


\begin{tabular}{cccccc}
\hline \#workers & pre & dec & merge & total \\
\hline 1 & 423 & 419 & 608 & 1450 \\
2 & 224 & 236 & 417 & 876 \\
4 & 121 & 123 & 301 & 545 \\
8 & 62 & 73 & 238 & 372 \\
16 & 40 & 35 & 237 & 312 \\
\hline
\end{tabular}

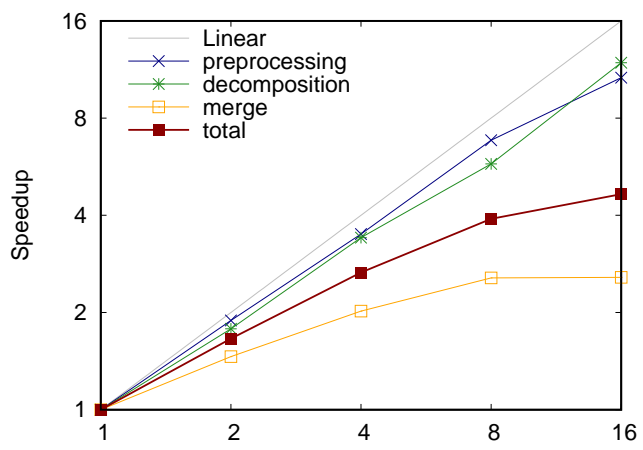

Fig. 8: Execution times (left) in seconds and speedup (right) for music dataset DS4 for the single workflow phases and total workflow.

Scalability To evaluate the distributed holistic clustering w.r.t. efficiency and scalability, we determine the absolute execution times as well as the speedup for the very large geographic (DS2) and music datasets (DS4, DS5). In Flink, several options allow the fine-tuning of parameters for a distributed cluster environment. In most cases the reasonable way to improve execution times for very large datasets is the increase of deployed workers. Another important parameter is the parallelism to specify the maximum number of parallel instances of operators or data sinks/sources that are available to process data within a Flink workflow. We here used the parallelism equal to the number of workers, for which we achieved the best execution times.

Fig. 7 and Fig. 8 show the achieved execution times for DS2 and DS4, respectively, for different phases of the clustering workflow as well as the overall workflow execution time. For each phase, an increased number of workers leads to improved execution times. For both domains, the best improvement can be achieved for the preprocessing (pre) and decomposition (dec) phases. The merge phase is by far more complex. While preprocessing and decomposition operate within connected components and clusters, the merge phase attempts to combine similar clusters based on the assignment in the blocking step and therefore can suffer from data skew problems for some blocks. These effects become also clear in Fig. 7 and Fig. 8 showing the speedup results compared to the linear optimum. Preprocessing and decomposition achieve nearly linear speedup, while the merge phase shows decreased speedup values. In total, we achieve a good speedup of 5.86 for the large geographic dataset DS2 and 4.65 for the large music dataset DS4. For the largest dataset DS5 with $\approx 20$ million entities, we could determine results for two configurations: 8 workers could finish the complex task in 43,589 seconds, and 16 workers finished after 24,722 seconds (reduced by factor $\approx 1.8$ ).

Overall, the distributed holistic clustering achieves good execution times and moderate scalability results for very large entity sets. The approach is scalable for different data sources and employs a multi-source clustering instead of basic binary linking of two sources. The distributed implementation further allows to scale for a growing number of entities and data sources and is very useful for complex data integration scenarios in big data processing workflows. 


\section{Related Work}

Link discovery (LD) has been widely investigated and there are many approaches and prototypes available as surveyed in [16]. Typical LD approaches apply binary linking methods for matching two data sources but lack efficient and effective methods for integrating entities from $k$ different data sources to provide a holistic view for linked data. Some approaches enable distributed link discovery or for matching two data sources. For instance, Silk MapReduce [10] and Limes [9] realized LD approaches based on MapReduce before distributed data processing frameworks like Spark or Flink became state of the art. Similarly, the entity resolution framework Dedoop [11] allows to execute complex matching workflows on MapReduce. These tools suffer from limitations of MapReduce like repeated data materialization within and between single jobs and the lack of iterations. They further focus on pairwise matching and do not support holistic clustering for multiple data sources or the reuse of existing links sets.

While LD is driven by pairwise linking of data sources, support for multiple data sources can be found in related research areas. In [6] ontology concepts from multiple data sources are clustered based on topic forests for extracted keywords from concepts and their descriptions to determine matching concepts within groups of similar topics. In [13] a maximum-weighted graph matching and structural similarity computations are applied to concepts of multiple ontologies to find high quality alignments. However, these holistic ontology matching approaches do not focus on clustering of concepts or entities and have limitations w.r.t. scalability for $\mathrm{LD}$ and many large entity data sources.

There are few LD approaches that apply clustering or linking for linked data on multiple sources. Thalhammer et al. [24] present a pipeline for web data fusion using multiple data sources applying hierarchical clustering, cluster refinement and selection of representatives to achieve a similarity-based clustering with unified entities. The unsupervised LD approach Colibri [18] considers error detection and correction for link discovery in multiple knowledge bases based on the transitivity of interlinked entities, while clustering of entities is not the main focus. These approaches do not realize distributed clustering or linking and have not been evaluated w.r.t. scalability. There has been some research for distributed clustering methods such as community detection within social networks, e. g., using MapReduce [14] or MapReduce and Spark [7]. However these approaches do not focus on complex link discovery and data integration workflows, and partially suffer from MapReduce limitations. The work in [23] considers the implementation of existing clustering algorithms on top of Apache Flink for entity resolution and de-duplication of several data sources. The approach does not handle incorrect links and does no use semantic type information. It further does not create representatives for a compact cluster representation as useful for further applications.

In own previous work [15], we proposed a holistic clustering approach for multiple Linked Data sources with few initial evaluation results. Here we present an extended workflow implementation, in particular the realization of holistic clustering in a distributed environment, and show comprehensive evaluation results w.r.t. to cluster quality based on a novel gold standard, as well as scalability for datasets covering very large entity sets from multiple different data sources and domains. The created compact cluster representation is particularly useful for reuse and incremental cluster extension. 


\section{Conclusion}

We presented a distributed holistic clustering workflow for linked data using the distributed data processing framework Apache Flink. Our approach is based on the reuse of existing links and is able to handle entities from various different data sources. We showed the realization of a complex holistic clustering workflow with dataset transformations and user-defined functions. Albeit based on Apache Flink, the approach could be also implemented on other frameworks for distributed data processing such as Apache Spark. We further provide a novel gold standard for multi-source clustering from the geographic domain to support the development and evaluation of novel holistic clustering methods. evaluation results for datasets from two different domains with up to 20 million entities. Our results showed that the proposed approach can achieve a very high cluster quality. In particular, we were able to find many new correct links and could remove wrong links within our clustering workflow. We further presented results for a distributed execution of the holistic clustering in a parallel cluster environment with very good execution times for the considered dataset sizes as well as good overall scalability results.

For future work, we plan to further improve the scalability of our approach, e.g., by realizing sophisticated blocking and load balancing methods for the complex cluster merge phase. We further plan the development and combination with an incremental clustering to support the addition of new entities and data sources and to particularly address the ongoing growth of the Web of Data and the accompanied need for incremental holistic clustering methods for LinkedData.

\section{Acknowledgments}

This research was supported by the Deutsche Forschungsgemeinschaft (DFG) grant number RA 497/19-2.

\section{References}

1. Aslam, J.A., Pelekhov, E., Rus, D.: The Star Clustering Algorithm for Static and Dynamic Information Organization. J. Graph Algorithms Appl. 8, 95-129 (2004)

2. Carbone, P., Katsifodimos, A., Ewen, S., Markl, V., Haridi, S., Tzoumas, K.: Apache Flink $^{\mathrm{TM}}$ : Stream and Batch Processing in a Single Engine. IEEE Data Eng. Bull. 38(4) (2015)

3. Dong, X., Gabrilovich, E., Heitz, G., Horn, W., Lao, N., Murphy, K., Strohmann, T., Sun, S., Zhang, W.: Knowledge Vault: A Web-Scale Approach to Probabilistic Knowledge Fusion. In: SIGKDD Int. Conference on Knowledge Discovery and Data Mining. pp. 601-610 (2014)

4. Faria, D., Jiménez-Ruiz, E., Pesquita, C., Santos, E., Couto, F.M.: Towards Annotating Potential Incoherences in BioPortal Mappings. In: ISWC. pp. 17-32 (2014)

5. Gonzalez, J.E., Low, Y., Gu, H., Bickson, D., Guestrin, C.: PowerGraph: Distributed GraphParallel Computation on Natural Graphs. In: Thekkath, C., Vahdat, A. (eds.) OSDI, Hollywood, CA, USA. pp. 17-30. USENIX Association (2012)

6. Grütze, T., Böhm, C., Naumann, F.: Holistic and Scalable Ontology Alignment for Linked Open Data. In: WWW2012 Workshop on Linked Data on the Web (2012) 
7. Guo, K., Guo, W., Chen, Y., Qiu, Q., Zhang, Q.: Community discovery by propagating local and global information based on the MapReduce model. Information Sciences 323 (2015)

8. Hildebrandt, K., Panse, F., Wilcke, N., Ritter, N.: Large-Scale Data Pollution with Apache Spark. IEEE Transactions on Big Data PP(99), 1-1 (2017)

9. Hillner, S., Ngonga Ngomo, A.C.: Parallelizing LIMES for large-scale link discovery. In: I-Semantics '11. pp. 9-16. ACM, New York, NY, USA (2011)

10. Isele, R., Jentzsch, A., Bizer, C.: Silk Server - Adding missing Links while consuming Linked Data. In: Proc. of the First Int. Workshop on Consuming Linked Data. CEUR Workshop Proc., vol. 665. CEUR-WS.org (2010)

11. Kolb, L., Thor, A., Rahm, E.: Dedoop: Efficient Deduplication with Hadoop. Proc. of the VLDB Endowment 5(12), 1878-1881 (2012)

12. Malewicz, G., Austern, M.H., Bik, A.J., Dehnert, J.C., Horn, I., Leiser, N., Czajkowski, G.: Pregel: A System for Large-scale Graph Processing. In: Proc. of the 2010 ACM SIGMOD Int. Conference on Management of Data. pp. 135-146. ACM (2010)

13. Megdiche, I., Teste, O., dos Santos, C.T.: An Extensible Linear Approach for Holistic Ontology Matching. In: The Semantic Web - ISWC 2016 - 15th Int. Semantic Web Conference. LNCS, vol. 9981, pp. 393-410 (2016)

14. Moon, S., Lee, J.G., Kang, M., Choy, M., woo Lee, J.: Parallel community detection on large graphs with MapReduce and GraphChi. Data \& Knowledge Engineering 104, 17 - 31 (2016)

15. Nentwig, M., Groß, A., Rahm, E.: Holistic Entity Clustering for Linked Data. In: Proc. ICDM Workshops. pp. 194-201. IEEE (2016)

16. Nentwig, M., Hartung, M., Ngomo, A.N., Rahm, E.: A survey of current Link Discovery frameworks. Semantic Web 8(3), 419-436 (2017)

17. Nentwig, M., Soru, T., Ngomo, A.C.N., Rahm, E.: LinkLion: A Link Repository for the Web of Data. In: The Semantic Web: ESWC 2014 Satellite Events - ESWC 2014 Satellite Events. LNCS, vol. 8798, pp. 439-443. Springer (2014)

18. Ngomo, A.N., Sherif, M.A., Lyko, K.: Unsupervised Link Discovery through Knowledge Base Repair. In: The Semantic Web: Trends and Challenges - 11th Int. Conference, ESWC 2014, Anissaras, Crete, Greece, May 25-29, 2014. Proc., pp. 380-394 (2014)

19. Ngonga Ngomo, A.C., Auer, S.: LIMES - A Time-Efficient Approach for Large-Scale Link Discovery on the Web of Data. In: Proc. of the 21. Int. Joint Conference on Artificial Intelligence. pp. 2312-2317. IJCAI'11, AAAI Press (2011)

20. Nickel, M., Murphy, K., Tresp, V., Gabrilovich, E.: A Review of Relational Machine Learning for Knowledge Graphs. Proc. of the IEEE 104(1), 11-33 (2016)

21. Noy, N.F., Shah, N.H., Whetzel, P.L., Dai, B., Dorf, M., Griffith, N., Jonquet, C., Rubin, D.L., Storey, M.D., Chute, C.G., Musen, M.A.: BioPortal: ontologies and integrated data resources at the click of a mouse. Nucleic Acids Research 37 (2009)

22. Rahm, E.: The Case for Holistic Data Integration. In: Advances in Databases and Information Systems - 20th East European Conference, ADBIS 2016, Prague, Czech Republic, August 28-31, 2016, Proceedings. pp. 11-27 (2016)

23. Saeedi, A., Peukert, E., Rahm, E.: Comparative Evaluation of Distributed Clustering Schemes for Multi-source Entity Resolution. In: Advances in Databases and Information Systems - 21th East European Conference, ADBIS 2017. LNCS (2017)

24. Thalhammer, A., Thoma, S., Harth, A., Studer, R.: Entity-centric Data Fusion on the Web. In: Proc. of the 28th ACM Conference on Hypertext and Social Media. ACM (2017)

25. Zaharia, M., Chowdhury, M., Das, T., Dave, A., Ma, J., McCauly, M., Franklin, M.J., Shenker, S., Stoica, I.: Resilient Distributed Datasets: A Fault-Tolerant Abstraction for InMemory Cluster Computing. In: NSDI, San Jose, CA, USA. pp. 15-28 (2012) 